\title{
BMJ Open Assisted reproductive technology and risk of asthma and allergy in the offspring: protocol for a systematic review and meta-analysis
}

\author{
Bright I Nwaru, ${ }^{1,2}$ Nicola McCleary, ${ }^{2}$ Maijaliisa Erkkola, ${ }^{3}$ Minna Kaila, ${ }^{4}$ \\ Suvi M Virtanen, ${ }^{5,6,7,1}$ Aziz Sheikh ${ }^{2}$
}

To cite: Nwaru $\mathrm{BI}$ McCleary N, Erkkola M, et al. Assisted reproductive technology and risk of asthma and allergy in the offspring: protocol for a systematic review and metaanalysis. BMJ Open 2016;6: e010697. doi:10.1136/ bmjopen-2015-010697

- Prepublication history and additional material is available. To view please visit the journal (http://dx.doi.org/ 10.1136/bmjopen-2015010697).

Received 27 November 2015 Revised 22 January 2016 Accepted 11 February 2016

CrossMark

For numbered affiliations see end of article.

Correspondence to Bright I Nwaru; bright. nwaru@uta.fi

\section{ABSTRACT}

Introduction: The use of assisted reproductive technology (ART) procedures has increased globally over the last three decades. Recent observational studies suggest that children conceived through ART may be at increased risk of asthma and atopic disease compared with children conceived naturally, but findings are mixed. We aim to synthesise the evidence on the impact of ART on the risk of asthma and atopic disease in the offspring. Methods and analysis: We will identify relevant studies by searching MEDLINE, EMBASE, Cochrane Library, ISI Web of Science, CINAHL, Scopus, Google Scholar, AMED, Global Health, PsychINFO, CAB International and the WHO Global Health Library from 1978 to 2016. We will locate additional studies through searching databases of the proceedings of international conferences, contacting international experts in the field, and searching the references cited in identified studies. We will include analytic observational studies (cohort studies, case-control studies and cross-sectional studies) that have investigated the impact of any type of ART on offspring's asthma and atopic disease. Screening of identified records, data extraction from eligible studies and risk of bias assessment of eligible studies will be independently undertaken by two reviewers, with arbitration by a third reviewer. The Effective Public Health Practice Project will be employed for risk of bias assessment. Estimates from studies judged to be clinically, methodologically and statistically homogeneous will be synthesised using random-effects meta-analysis.

Ethics and dissemination: As this study is based solely on the published literature, no ethics approval is required. We will publish our findings in a peer-reviewed scientific journal and present the results at national and international scientific conferences.

Protocol registration: We will register a detailed protocol for the review with the International Prospective Register of Systematic Reviews (PROSPERO) prior to starting the review.

\section{INTRODUCTION}

Since its inception in 1978, the use of assisted reproductive technology (ART) has

\section{Strengths and limitations of this study}

- As the use of assisted reproductive technology becomes more common, clarifying its impact on disease risk in offspring, such as risk of asthma and allergy, is essential for decision-making.

- This is the first systematic review of the impact of assisted reproductive technology on asthma and allergy in offspring, and it will provide a comprehensive synthesis of the underlying evidence base.

- The identification of studies from leading medical and public health databases, with no geographical or language limitations, will advance import of this evidence synthesis across settings.

dramatically increased globally. ${ }^{1-6}$ It is now estimated that ART accounts for between 1\% and $4 \%$ of all births, particularly in industrialised societies, but anecdotal data suggest that its use is rising in low-income and middle-income countries as well. ${ }^{1-6}$ Until recently, in vitro fertilisation constituted the majority of ART methods, but the use of intracytoplasmic sperm injection has steadily increased in recent times, and is now believed to comprise up to $70 \%$ of all ART procedures; the use of other procedures, such as fresh and frozen embryo transfers and intrauterine insemination, is steadily increasing. ${ }^{12}$

Over the years, there have been concerns about the short-term and long-term risks for children conceived through ART compared with those for naturally conceived children. ${ }^{127}$ Children conceived through ART are believed to phenotypically and biochemically differ from those conceived naturally, but the mechanisms underlying these differences and the subsequent health implications are unclear. $^{2}$ Amidst conflicting 
findings, some studies have suggested that ART children are at increased risk of key perinatal outcomes, including congenital malformations, prematurity, low birth weight, hypertensive disease, diabetes, perinatal mortality, imprinting disorders and certain cancers. ${ }^{1-6}$ However, some investigators suggest that these observations may be a consequence of potential biases inherent in observational epidemiological studies, underlying maternal factors such as subfertility, age and parity, or a combination of these factors and ART, and not necessarily the ART procedure alone. ${ }^{127}$

Recently, some studies have investigated the relationship between ART and the risk of asthma and atopic disorders in children conceived through ART compared with that in children conceived naturally, but findings are conflicting. ${ }^{8-15}$ While the possible biological mechanism for these associations, as in other perinatal outcomes, has not been clearly addressed, some argue that the observed associations may be attributed to maternal subfertility, residual confounding, or other immune modifying maternal factors during pregnancy, such as pre-existing conditions including but not limited to asthma and allergy, or other extrinsic factors such as medications and smoking. ${ }^{16}$ Furthermore, it has been suggested that, since women undergoing ART procedures are generally of higher socioeconomic status, and have higher body mass with increased prevalence of metabolic disorders, their offspring may be at an increased risk of adverse outcomes. ${ }^{16}$ The high prevalence of metabolic impairment in the infertile patient population may therefore have long-term transgenerational impact, either through genetic or epigenetic mechanisms as a result of embryo culture and the potency of the fertility drugs used for treating resultant ovarian hyperstimulation. ${ }^{16} 17$

Given the increasing number of studies relating ART to asthma and atopic disease in the offspring and mixed findings now being observed, a comprehensive synthesis of these studies is essential in order to clearly appreciate the underlying evidence relating ART to the aetiology and outcomes of asthma and atopic disease in the offspring. A synthesis of the evidence base will also help to identify relevant gaps in research in this area and suggest key steps in addressing these gaps. Therefore, in this study, we aim to identify, critically appraise and synthesise the evidence on the use of ART, and the risk of asthma and atopic disease in the offspring.

\section{METHODS}

We have followed the recommendations of the Preferred Reporting Items for Systematic review and Meta-Analysis Protocols (PRISMA-P) checklist in reporting this protocol. ${ }^{18}$

\section{Eligibility criteria}

Types of studies

We will include all analytic observational epidemiological studies (cohort studies; case-control studies; and cross-sectional studies) that have been conducted on the topic. We will exclude reviews, case studies and case series and animal studies.

\section{Participants}

Eligible participants will include women with evidence of conception history and their offspring of any age.

\section{Years considered}

Given that the first ART procedure was undertaken in $1978,{ }^{1}{ }^{2}$ we will consider all evidence emanating from this date up to 2016.

\section{Language}

There will be no language restrictions and, where possible, we will translate the literature published in languages other than English.

\section{Information sources}

Database searches and other sources to identify studies

We will search MEDLINE, EMBASE, Cochrane Library, ISI Web of Science, CINAHL, Scopus, Google Scholar, AMED, Global Health, PsychINFO, CAB International and the WHO Global Health Library. The databases will be searched for studies indexed from 1978 until 2016. We will locate additional references through searching the references cited in identified studies; through searching databases of the proceedings of international conferences, such as ISI Conference Proceedings Citation Index via Web of Knowledge, ZETOC (British Library); and by contacting a panel of international experts and authors who have published in the field. We will search trial registries, such as Current Controlled Trials (http://www.controlled-trials.com), ClinicalTrials. gov (http://www.clinicaltrials.gov) and Australian and New Zealand Clinical Trials Registry (http://www.anzctr. org.au), to identify ongoing studies.

\section{Search strategy}

Using the Ovid interface for MEDLINE, we have developed a highly sensitive and comprehensive search strategy (see online supplementary appendix 1) to identify and retrieve relevant and eligible studies. This search strategy will be adapted in searching the other databases.

\section{Study records}

\section{Data management}

The retrieved records from all databases will be exported to Endnote Library, which will be used throughout the review for study screening, deduplication and overall management of the retrieved records.

\section{Selection process}

Titles and abstracts of retrieved articles will be screened and full text copies of potentially eligible studies assessed by two independent reviewers; a third reviewer will arbitrate any discrepancies. Studies that do not fulfil the inclusion criteria will be excluded. 


\section{Data extraction}

Two reviewers will independently extract relevant study data from eligible studies onto a customised data extraction form; a third reviewer will arbitrate any discrepancies. Before using the form for all studies, we will pilot the data extraction form with a selected sample of studies in order to evaluate the ability of the form to capture the relevant study data of interest.

\section{Data items}

Descriptive summary tables will be produced to summarise the literature and we will tabulate all relevant study data. In addition to other relevant study data as may be available from each study, we aim to capture, as a minimum, the following data items from each study: study author; country of study; year of publication; type of study design; study size; source of study population; type of ART (and comparison group) and method of assessment; singleton versus multiple pregnancy; length of follow-up (for follow-up studies); key potential confounders (maternal age, parity, subfertility, history of asthma/ allergy, maternal smoking during pregnancy and sex of child); study outcomes and methods of assessment; analysis methods; and key results. The PRISMA checklist will guide the reporting of the systematic review. ${ }^{19}$

\section{Types of exposures}

We will include all studies that have investigated the role of any type of ART (in vitro fertilisation, intrauterine insemination, intracytoplasmic sperm injection, zygote intrafallopian transfer, gamete intrafallopian transfer, medicinal and surgical infertility treatments) in comparison with natural conception or any other comparison group as reported in the studies.

\section{Outcomes and prioritisation}

Our primary outcomes will include: objectively measured or self-reported asthma, atopic dermatitis/eczema, allergic rhinitis, anaphylaxis, urticaria, angio-oedema and food allergy. The secondary outcomes will include: atopic sensitisation as defined either by skin prick test or raised antigenspecific IgE; objective and subjective measures of disease severity and impact on quality of life, including asthma exacerbations, use of asthma medications, hospitalisation for asthma, wheeze as defined by self-report or objective diagnosis; indicators of airway function (including peak expiratory flow, forced expiratory volume in $1 \mathrm{~s}$, forced vital capacity, forced expiratory flow rate or alternative age appropriate pulmonary function tests (oscillometry or exhaled nitric oxide analysis)); and measures of patient-reported health-related quality of life related to asthma or allergy.

\section{Risk of bias in individual studies}

Risk of bias in eligible studies will be assessed by two reviewers; a third reviewer will arbitrate any discrepancies. We will assess the risk of bias by using the Effective Public Health Practice Project (EPHPP) tool (http:// www.ephpp.ca). We will grade the following components of each study: suitability of the study design for the research question; risk of selection bias; exposure measurement; outcome assessment; and generalisability of findings. From these component-specific assessments, we will derive an overall grading for each study.

\section{Data synthesis}

To summarise the overall evidence, we will undertake a narrative synthesis of the data. Additionally, for clinically, methodologically and statistically homogeneous studies, we will perform meta-analyses using random-effects models to quantify a pooled estimate of the effect of specific types of ART on the risk of asthma and atopic disease in the offspring. Meta-analyses will be undertaken separately for each specific study design. In comparison with fixed-effect meta-analysis, using random-effects models to compute the pooled estimates presents a more conservative option, as the underlying assumption of random-effects meta-analysis of non-common effect across studies is more realistic when involving studies obtained solely from the published literature. ${ }^{20}$ The random-effects model also takes into account potential heterogeneity between studies when computing the pooled estimates. ${ }^{20}$ We will quantify the heterogeneity between studies using the $\mathrm{I}^{2}$ statistic. We will undertake the following subgroup analyses: by age of offspring at onset/diagnosis of outcomes (where possible using the following age groups: <5 years, 5-12 years, >12 years); singleton versus multiple pregnancy; single versus double embryo transfers; parity; and length of subfertility. We will undertake a sensitivity analysis by the grading of study quality in order to evaluate the robustness of our findings. The meta-analyses will be performed using the Stata 14 statistical package (StataCorp. 2015. Stata Statistical Software: Release 14. College Station, TX: StataCorp LP).

\section{Publication bias}

We will evaluate the potential for publication bias by using funnel plots and Begg and Egger tests. ${ }^{21} 22$

\section{Protocol registration}

A detailed protocol for the review is registered with the International Prospective Register of Systematic Reviews (PROSPERO): 2016:CRD42016035966 (http:// www.crd.york.ac.uk/PROSPERO/display_record.asp? $\mathrm{ID}=\mathrm{CRD} 42016035966$ ).

\section{Confidence in the cumulative estimate}

We will evaluate the strength of the overall evidence through assessment of the clinical and methodological heterogeneity across studies and on the basis of the risk of bias assessment in included studies. We will consider these lines of impact on the overall evidence in reaching a conclusion on the import of findings and in recommending for future direction in the field. Furthermore, we will grade the strength and quality of the overall evidence by using the Grading of Recommendations Assessment, Development and Evaluation (GRADE) approach. ${ }^{23}$ 


\section{CONCLUSION}

The increasing use of ART and its potential implication for increased risk of asthma and atopic disease in the offspring now requires a comprehensive evidence synthesis, which will provide us with the opportunity to appreciate the underlying evidence base and assess its policy, practice and public health implications. In addition, this evidence synthesis provides the opportunity to identify the research gaps in studies linking ART to the development of asthma and atopic disease in the offspring. We aim to report the findings from this review by autumn 2016.

\section{Author affiliations}

${ }^{1}$ School of Health Sciences, University of Tampere, Tampere, Finland

${ }^{2}$ Asthma UK Centre for Applied Research, Centre for Medical Informatics, Usher Institute of Population Health Sciences and Informatics, The University of Edinburgh, Edinburgh, UK

${ }^{3}$ Department of Food and Environmental Sciences, University of Helsinki, Helsinki, Finland

${ }^{4}$ Department of Public Health, University of Helsinki, Helsinki, Finland

${ }^{5}$ Nutrition Unit, Department of Lifestyle and Participation, National Institute for Health and Welfare, Helsinki, Finland

${ }^{6}$ Tampere Centre for Child Health Research, Tampere University Hospital, Tampere, Finland

${ }^{7}$ Science Centre of Pirkanmaa Hospital District, Tampere University Hospital and University of Tampere, Tampere, Finland

Contributors BIN conceived the idea for this work and is the guarantor. AS contributed subject expertise to the development of the protocol. The protocol was drafted by BIN and then revised after several rounds of critical comments from AS and additional feedback from ME, MK, SMV and NM. All the authors will be involved in the systematic review process.

Funding This work is funded by a fellowship award to BIN from the Institute for Advanced Social Research, with additional support from the School of Health Sciences, University of Tampere, Finland. Additional support was also provided by the Asthma UK Centre for Applied Research, The University of Edinburgh, UK.

Disclaimer The views presented here are those of the authors and not necessarily those of the Universities of Tampere and Edinburgh.

Competing interests None declared

Provenance and peer review Not commissioned; externally peer reviewed.

Open Access This is an Open Access article distributed in accordance with the Creative Commons Attribution Non Commercial (CC BY-NC 4.0) license, which permits others to distribute, remix, adapt, build upon this work noncommercially, and license their derivative works on different terms, provided the original work is properly cited and the use is non-commercial. See: http:// creativecommons.org/licenses/by-nc/4.0/

\section{REFERENCES}

1. Talaulikar VS, Arulkumaran S. Reproductive outcomes after assisted conception. Obstet Gynecol Surv 2012;67:566-83.

2. Savage T, Peek J, Hofman PL, et al. Childhood outcomes of assisted reproductive technology. Hum Reprod 2011;26:2392-400.

3. de Mouzon J, Goossens V, Bhattacharya S, et al. Assisted reproductive technology in Europe, 2006: results generated from European register by ESHRE. Hum Reprod 2010;25:1851-62.

4. Basatemur E, Sutcliffe A. Follow-up of children born after ART. Placenta 2008;29:S135-40.

5. Schieve LA, Ferre C, Peterson HB, et al. Perinatal outcome among singleton infants conceived through assisted reproductive technology in the United States. Obstet Gynecol 2004;103:1144-53.

6. Schieve LA, Rasmussen SA, Buck GM, et al. Are children born after assisted reproductive technology at increased risk for adverse health outcomes? Obstet Gynecol 2004;103:1154-63.

7. Lambert RD. Safety issues in assisted reproductive technology: aetiology of health problems in singleton ART babies. Hum Reprod 2003;18:1987-91.

8. Jäderberg I, Thomsen SF, Kyvik KO, et al. Atopic diseases in twins born after assisted reproduction. Paediatr Perinat Epidemiol 2012;26:140-5.

9. Guibas GV, Moschonis G, Xepapadaki P, et al. PD44-In vitro fertilisation is positively associated with prevalence of asthma in childhood. Clin Transl Allergy 2014:4:P44.

10. Sicignano N, Beydoun HA, Russell $\mathrm{H}$, et al. A descriptive study of asthma in young adults conceived by IVF. Reprod Biomed Online 2010;21:812-18.

11. Carson C, Sacker A, Kelly Y, et al. Asthma in children born after infertility treatment: findings from the UK Millennium Cohort Study. Hum Reprod 2013:28:471-9.

12. Källén B, Finnström O, Nygren KG, et al. Asthma in Swedish children conceived by in vitro fertilisation. Arch Dis Child 2013;98:92-6.

13. Guibas GV, Moschonis G, Xepapadaki P, et al. Conception via in vitro fertilization and delivery by Caesarean section are associated with paediatric asthma incidence. Clin Exp Allergy 2013;43:1058-66.

14. Cetinkaya F, Gelen SA, Kervancioglu E, et al. Prevalence of asthma and other allergic diseases in children born after in vitro fertilisation. Allergol Immunopathol 2009;37:11-3.

15. Klemetti R, Sevón T, Gissler M, et al. Health of children born as a result of in vitro fertilization. Pediatrics 2006;118:1819-27.

16. Davies MJ. Infertility treatment at the edge: discovery and risk converge at the limits of knowledge. Arch Dis Child 2013;98:89-90.

17. Shiota K, Yamada S. Intrauterine environment-genome interaction and children's development (3): assisted reproductive technologies and developmental disorders.J Toxicol Sci 2009;34(Suppl 2): SP287-91.

18. Shamseer L, Moher D, Clarke M, et al. Preferred reporting items for systematic review and meta-analysis protocols (PRISMA-P) 2015: elaboration and explanation. BMJ 2015;349:g7647.

19. Moher D, Liberati A, Tetzlaff $J$, et al. The PRISMA Group. Preferred reporting items for systematic reviews and meta-analyses: the PRISMA statement. PLoS Med 2009;6:e1000097.

20. Borenstein M, Hedges LV, Higgins JPT, et al. A basic introduction to fixed-effect and random-effects models for meta-analysis. Res Synth Methods 2010;1:97-111.

21. Begg CB, Mazumdar M. Operating characteristics of a rank correlation test for publication bias. Biometrics 1994;50:1088-101.

22. Egger M, Smith GD, Schneider M, et al. Bias in meta-analysis detected by a simple, graphical test. BMJ 1997;315:629-34.

23. Guyatt GH, Oxman AD, Vist GE, et al. GRADE: an emerging consensus on rating quality of evidence and strength of recommendations. BMJ 2008;336:924-6. 